\title{
Advanced Feedback Encryption Standard Version-1 (AFES-1)
}

\author{
Debdeep Basu \\ Department of Computer \\ Science \\ St. Xavier's \\ College(Autonomous) \\ Kolkata, India
}

\author{
Ankita Bose \\ Department of Computer \\ Science \\ St. Xavier's \\ College(Autonomous) \\ Kolkata, India
}

\author{
Surajit Bhowmik \\ Department of Computer \\ Science \\ St. Xavier's \\ College(Autonomous) \\ Kolkata, India
}

\author{
Saptarshi Chatterjee \\ Department of Computer \\ Science \\ St. Xavier's \\ College(Autonomous) \\ Kolkata, India
}

\author{
Asoke Nath \\ Department of Computer \\ Science \\ St. Xavier's \\ College(Autonomous) \\ Kolkata, India
}

\begin{abstract}
In this paper, Advanced Feedback Encryption Standard Version 1 (AFES-1), Nath et al have combined both bit-level and byte level operations on the plain text. Nath et al had recently published Multi Way Feedback Encryption Standard Ver-3 MWFES-3[5]. MWFES-3 is a byte level encryption algorithm. The authors have capitalized on the strength of MWFES-3[5] by introducing a bit-shuffling operation at the beginning of each iteration. At the beginning of each iteration, the plain text bits of that iteration are shuffled by using 24 different shuffling functions. Now, the order in which the 24 different functions are called, changes at each iteration, and that order is taken as a function of the key. After the initial shuffling of the bits, the bits are converted back to bytes and MWFES-3 is applied on the bytes. This process goes on Encryption Number (EN) times, where EN is also taken as a function of the key. So, at the beginning of each iteration, the bits obtained from the last iteration are shuffled in a different way. This method has been tested on standard plain texts such as ASCII ' 0 ', ASCII ' 1 ' and the results are quite satisfactory. This method is immune to any classical form of attacks.
\end{abstract}

\section{General Terms}

Encryption, Decryption.

\section{Keywords}

MWFES-I, MWFES-2, MWFES-3, Encryption Number.

\section{INTRODUCTION}

Due to the tremendous development in internet technologies it is essential to encrypt any kind of confidential message before sending the message from one computer to another computer. Data security is an extremely important issue and many algorithms have been developed which are almost impossible to break. The intention of the trespasser is to break the cipher and to retrieve unauthorized information. It is the job of the cryptographers to restrict the trespassers from achieving unauthorized access. Nath et al had recently proposed
MWFES-1[1], Modified MWFES-1[2], MWFES-2[3], Modified MWFES-2[4], MWFES-3[5].

In MWFES-1, the plain text character is added with the corresponding key character, the forward feedback and backward feedback and then the total sum (modulo 256) is taken as the corresponding cipher text character. The cipher text character is taken as the forward feedback value for the next byte (in case of forward operation) or backward feedback value for the previous byte (in case of backward operation). Forward and Backward operations are carried out on all the bytes starting from their respective ends.

In MWFES-2, the process is a little more general. Instead of propagating the feedback to the next byte (in case of forward feedback) and to the previous byte (in case of backward feedback) the feedback is propagated to the $n^{\text {th }}$ byte where $n$ is the 'skip factor'. In MWFES-2 the forward skip is kept equal to the backward skip (equal to n) and the initial forward feedback value and the backward feedback value was kept 0 .

In MWFES-3, the authors introduced several changes in the algorithm. The plain text is broken into blocks and the encryption method is applied on each block separately. Each block has different Forward Skip (FS), Backward Skip (BS), initial Forward Feedback (FF) and initial Backward Feedback (BF) which are determined from the keypad counterpart of the block. These four important variables would decide the nature of the cipher text. The block size is different in every round of processing, causing these four important variables to change in every round. The total number of rounds (encryption no), and the block size value were also taken as a function of the key.

In the present algorithm, i.e. AFES-1 the Plain Text is converted to its corresponding bits and stored in a square matrix of size equal to the integral square root of the number of bits. The residual bits remain untouched. Then the bits are arranged by calling 24 different shifting functions. Now, the order of calling the 24 functions change at each iteration and 
that order is taken as a function of the keypad. These 24 functions can be extended to ' $n$ ' functions as long as each of the functions is reversible. After this is done, the bits are converted back to bytes and then MWFES-3[5] is applied on those bytes. This entire process happens Encryption Number (EN) times. Therefore, in each iteration, the bits are permuted in a different way and then MWFES-3 is applied on the resultant bytes. Thorough tests were conducted on some standard plain text files and it was found that it is absolutely impossible for any intruder to extract any plain text from the generated encrypted text using any brute force method. The results show that the present method is free from any kind of classical attacks.

The present method is an extremely strong method as all controlling parameters change at every round. The present method may be applied in any Corporate sector, Defense sector, Government sector etc. The entire encryption and decryption software have been developed using MATLAB.

\section{ALGORITHM FOR AFES-1}

In the present section the encryption algorithm, key generation algorithm as well as decryption algorithm will be discussed.

\subsection{Algorithm for function encryption()}

Step 1: Start

Step 2: Input PlainText, User Provided Seed and Cipher Text filenames

Step 3: length=length(pt) $/ * \mathrm{pt}$ is the PlainText*/

Step 4: seed[]=Stores content of seed given by User

Step 5: $n=16$

Step 6: If $n * n<l e n g t h$, the go to Step 7 ,otherwise go to Step 8

Step 7: $\mathrm{n}=\mathrm{n}+1$ and go to Step 6

Step 8: key[]=Call key_generation(seed[],n)

Step 9: encryption_no=key[fix $((n * n) / 2)]$

Step 10: encryption_no=mod(encryption_no,64)

Step 11: if encryption_no $=0$ then encryption_no=1

Step 12: $\mathrm{e}=1$

Step 13: If e<=encryption_no then go to Step 14 otherwise go to Step 64

Step 14: Initialise sum,ff[length],bf[length],ct[length] with zeros $/ * \mathrm{ct}=$ CipherText,ff=Forward Feedbacks,bf=Backward Feedbacks*/.

Step 15: Call pt=pt_Shift(key[e]).

Step 16: block_size=key[e]

Step 17: If block_size>length, then go to Step 18, otherwise go to Step 19

Step 18: block_size=block_size-4

Step 19: If block_size $<4$,then block_size $=4$

Step 20: Initialise k, low and no_of_block with 1

Step 21: high=block_size

Step 22: If k>=block_size, go to Step 23,otherwise go to Step 36

Step 23: k=k-block_size

Step 24: forward_next=mod(key[low]+1,block_size)

Step 25: backward_next=mod(key[high]+1,block_size $)$
Step 26: If forward_next $=0$, then forward_next $=1$

Step 27: If backward_next $=0$, then backward_next $=1$

Step 28: forward_feedback=key[low+1]

Step 29: backward_feedback=key[high-1]

Step 30: ff[low]=forward_feedback

Step 31: bf[high]=backward_feedback

Step 32: Call encryption_block(low,high)

Step 33: low=high+1

Step 34: high=high+block_size

Step 35: no_of_block=no_of_block+1 and go to Step 22

Step 36: i=low

Step 37: If $i<=$ length, go to Step 38, otherwise go to Step 40

Step 38: $\mathrm{ct}[\mathrm{i}]=\mathrm{pt}[\mathrm{i}]$

Step 39: $i=i+1$ and go to Step 37

Step 40: If $k>0$, go to Step 41,otherwise go to Step 62

Step 41: i=length-k

Step 42: If $i>=1$,go to Step 43,otherwise go to Step 45

Step 43: $\mathrm{ct}[\mathrm{i}+\mathrm{k}]=\mathrm{ct}[\mathrm{i}]$

Step 44: i=i-1 and go to Step 42

Step 45: j=low

Step 46: $i=1$

Step 47: If $\mathrm{i}<=\mathrm{k}$,go to Step 48,otherwise go to Step 51

Step 48: ct[i] $=\mathrm{pt}[\mathrm{j}]$

Step 49: $\mathrm{j}=\mathrm{j}+1$

Step 50: $i=i+1$ and go to Step 47

Step 51: pt[]=ct[]

Step 52: forward_next=mod((key[1]+1),256)

Step 53: backward_next=mod((key[block_size]+1),256)

Step 54: If forward_next $=0$, then forward_next $=1$

Step 55: If backward_next $=0$, then backward_next $=1$

Step 56: forward_feedback=key[2]

Step 57: backward_feedback=key[block_size-1]

Step 58: Initialise ff[length] and bf[length] with zeros

Step 59: ff[1]=forward_feedback

Step 60: bf[block_size]=backward_feedback

Step 61: Call encryption_block(1,block_size)

Step 62: pt[]=ct[] /*Copying converted PlainText into CipherText array */

Step 63: e=e+1 and go to Step 13

Step 64: End

\subsection{Algorithm for function encryption_block (low,high)}

Step 1: Initialize $\mathrm{i}=$ low

Step 2: $\operatorname{sum}[\mathrm{i}]=\mathrm{pt}[\mathrm{i}]+\mathrm{key}[\mathrm{i}]+\mathrm{ff}[\mathrm{i}]+\mathrm{bf}[\mathrm{i}]$

Step 3: $\operatorname{ct}[\mathrm{i}]=\bmod (\operatorname{sum}[\mathrm{i}], 256)$;

Step 4: if i+forward_next $>$ high go to step 5,else go to step 6

Step 5: ff[low+(i+forward_next-high)-1]=ct[i]

Step 6: $f f[i+$ forward_next $]=\operatorname{ct}[i]$; 
Step 7: index=high-(i-low)

Step 8: $\operatorname{sum}[$ index $]=\mathrm{pt}[$ index $]+\operatorname{key}[$ index $]+\mathrm{ff}[$ index $]+$ bf[index]

Step 9: $\operatorname{ct}[$ index $]=\bmod (\operatorname{sum}($ index $), 256)$

Step 10: If index-backward_next $<$ low go to step 11 ,else go to step 12

Step 11: bf[high-(low-(index-backward_next))+1]=ct[index]

Step 12: Return control to the calling function

\subsection{Algorithm for function key_generation(seed[],n)}

From secret_key(seed) the program will generate an enlarged keypad. This keypad is stored in a square matrix having dimensions equal to the nearest greater perfect square of the Plain Text length. The first element of this keypad is taken to be sum of the ASCII codes of the characters in the seed. Now the next elements are simply values that are linearly multiplied with the sum, i.e $\mathrm{k}^{*}$ sum for $\mathrm{k}=1,2,3$ etc. If there is any repetition, then the program will modify the initial sum to be sum=sum +1 and the process will continue. The value of sum and its subsequent multiplications are kept within the boundaries of $0-255$ by applying the modulo operations on the numbers at every iteration and only after that the numbers are added to the keypad matrix. This way an initial matrix is created. The elements of the matrix were reshuffled using some simple operations such as upshift(), leftshift(),diagonal1_shift(),downshift(),rightshift(),diagonal2 _shift() etc. The final key was developed using various properties of the seed as well as intrinsic properties of the keypad. Any other appropriate key expansion algorithm can also be used for encryption and decryption.

Step 1: seed_l=length(seed)

Step 2: $\operatorname{sum}=0$

Step 3: i=1

Step 4: If $i<=$ seed_1,then go to Step 5,otherwise go to Step7

Step 5: sum=sum+seed[i]

Step 6: $i=i+1$ and go to Step 4

Step 7: $\operatorname{sum}=\bmod (\operatorname{sum}, 256)$

Step 8: Initialise array key[] with all zeros

Step 9: sum1=sum

Step 10: i=1

Step 11: If $\mathrm{i}<=$ n,go to Step 12,otherwise go to Step 21

Step 12: $\mathrm{j}=1$

Step 13: If $\mathrm{j}<=\mathrm{n}$,go to Step 14,otherwise go to Step 20

Step 14: $\operatorname{key}[\mathrm{i}][\mathrm{j}]=\bmod (\operatorname{sum} 1,256)$

Step 15: sum1=sum1+sum

Step 16: If $\bmod (\operatorname{sum} 1,256)=\bmod (\operatorname{sum}, 256)$,go to Step 17,otherwise go to Step 19

Step 17: $\operatorname{sum}=\bmod (\operatorname{sum}+1,256)$

Step 18: sum1=mod(sum,256)

Step 19: $\mathrm{j}=\mathrm{j}+1$ and go to Step 13

Step 20: $\mathrm{i}=\mathrm{i}+1$ and go to Step 11

Step 21: $i=1$

Step 22: If $i<=$ seed_l,go to Step 23,else go to Step 31

Step 23: key[][]=Call left_shift(key[][],n)
Step 24: key[][]=Call down_shift(key[][],n)

Step 25: key[][]=Call circular(key[][],n)

Step 26: key[][]=Call right_shift(key[][],n)

Step 27: key[][]=Call up_shift(key[][],n)

Step 28: key[][]=Call diagonal1_shift(key[][],n)

Step 29: key[][]=Call diagonal2_shift(key[][],n)

Step 30: $\mathrm{i}=\mathrm{i}+1$ and go to Step 22

Step 31: sum $=0$

Step 32: $\mathrm{i}=1$

Step 33: If $\mathrm{i}<=$, go to Step 34,otherwise go to Step 36

Step 34: sum=sum+key[i][i]

Step 35: sum=sum+key[i][n-i+1]

Step 36: randomization_number $=\bmod (\operatorname{sum}, 256)$

Step 37: i=1

Step 38: If i<=randomization_number,go to Step 39,else go to Step 47

Step 39: key[][]=Call left_shift(key[][],n)

Step 40: key[][]=Call down_shift(key[][],n)

Step 41: key[][]=Call circular(key[][],n)

Step 42: key[][]=Call right_shift(key[][],n)

Step 43: key[][]=Call up_shift(key[][],n)

Step 44: key[][]=Call diagonal1_shift(key[][],n)

Step 45: key[][]=Call diagonal2_shift(key[][],n)

Step 46: $i=i+1$ and go to Step 38

Step 47: Initialise array key1[] containing $(n * n)$ elements with all zeros

Step 48: $\mathrm{k}=1$

Step 49: $i=1$

Step 50: If $\mathrm{i}<=$, go to Step 51,otherwise go to Step 57

Step 51: $\mathrm{j}=1$

Step 52: If $\mathrm{j}<=$, go to Step 53,otherwise go to Step 56

Step 53: key1[k]=key[i][j]

Step 54: $\mathrm{k}=\mathrm{k}+1$

Step 55: $\mathrm{j}=\mathrm{j}+1$ and go to Step 52

Step 56: $\mathrm{i}=\mathrm{i}+1$ and go to Step 50

Step 57: Return array key1[] to calling function

\subsection{Algorithm for function decryption()}

Step 1: Start

Step 2: Input the CipherText,User Provided Seed and decrypted PlainText(Output) filenames.

Step 3: len=length(CipherText)

Step 4: seed[]=User Provided Key

Step 5: $\mathrm{n}=16$

Step 6: Is $(\mathrm{n} * \mathrm{n})<\operatorname{len}$ ?

Step 7: If Step $6=$ True, then $n=n+1$ and go to Step 6 .

Step 8: If Step 6=False, then go to Step 9

Step 9: key=Call key_generation(seed,n)

Step 10: encryption_no=key[fix $((n * n) / 2)]$

Step 11: e=encryption_no 
Step 12: block_size=key[e]

Step 13: Is block_size>len?

Step 14: If Step 12=True, then block_size=block_size-4 and go to Step 13.

Step 15: If Step 12=False,then go to Step 16.

Step 16: Is block_size<4?

Step 17: If Step 16=True, then block_size $=4$

Step 18: If Step 16=False, then go to Step 20.

Step 19: remainder=mod(len,block_size);

Step 20: initialise the array ct_temp[block_size] with all zeros;

Step 21: initialise the array key_temp[block_size] with all zeros;

Step 22: Is remainder! $=0$ ?

Step 23: If Step 22=True, go to Step 24,else go to Step 44.

Step 24: $\mathrm{t}=1$

Step 25: ct_temp $[\mathrm{t}]=\mathrm{ct}[\mathrm{t}]$

Step 26: key_temp $[\mathrm{t}]=\mathrm{key}[\mathrm{t}]$

Step 27: If $\mathrm{t}>$ block_size, go to step 28 ,else $\mathrm{t}=\mathrm{t}+1$ and go to Step 25.

Step 28: forward_next=key_temp[1]

Step 29: backward_next=key_temp[block_size]

Step 30: forward_feedback=key_temp[2]

Step 31: backward_feedback=key_temp[block_size-1]

Step32: pt=Call decryption_block (ct_temp, key_temp forward_next,backward_next,forward_feedback,backward_fe edback,block_size)

Step 33: j=len-remainder+1

Step 34: i=1

Step 35: pt_main[j]=pt[i]

Step 36: $\mathrm{j}=\mathrm{j}+1$

Step 37: If $i>$ remainder, then go to Step 38 ,else $i=i+1$ and go to Step 35 .

Step 38: i=1

Step 39: $\mathrm{ct}[\mathrm{i}]=\mathrm{pt}[\mathrm{i}]$

Step 40: If $i>$ block_size, then go to Step 41 else $i=i+1$ go to Step 39.

Step 41: i=remainder+1

Step 42: $c t[i-$ remainder $]=c t[i]$

Step 43: If $i>l e n$,then go to Step 44 ,else $i=i+1$ and go to Step 42.

Step 44: begin $=1$

Step 45: tot_div=floor(len/block_size)

Step 46: i=1

Step 47: set all elements of ct_temp[block_size] by zero

Step 48: set all key_temp[block_size] with all zeros

Step 49: $\mathrm{j}=1$

Step 50: $\mathrm{t}=$ =begin

Step 51: ct_temp[j] $=$ ct $[\mathrm{t}]$

Step 52: key_temp[j] $=k e y[t]$

Step 53: $\mathrm{j}=\mathrm{j}+1$
Step 54: If $\mathrm{t}>$ begin+block_size-1 then go to Step 55 else $\mathrm{t}=\mathrm{t}+1$ , go to Step 51 .

Step 55: forward_next=key_temp[1]

Step 56: backward_next=key_temp[block_size]

Step 57: forward_feedback=key_temp[2]

Step 58: backward_feedback=key_temp[block_size-1]

Step59: $\mathrm{pt}=$ Call decryption block (ct temp, key temp, forward_next,backward_next,forward_feedback,bacward_fee dback,block_size)

Step 60: $\mathrm{j}=1$

Step 61: k=begin

Step 62: pt_main[k]=pt[j]

Step 63: $j=j+1$

Step 64: If k>begin+block_size-1, then go to Step 65,else $\mathrm{k}=\mathrm{k}+1$ and go to Step 62 .

Step 65: begin=begin+block_size

Step 66: If $\mathrm{i}>$ tot_div,then go to Step 67 ,else $\mathrm{i}=\mathrm{i}+1$ and go to Step 46.

Step 67: ct[]=pt_main[]

Step 68: ct=Call ct_shift(pt_main[],key[e])

Step 69: If $\mathrm{e}<1$,then go to Step 70,else e=e-1 and go to Step 12.

Step 70: Write contents of pt_main into output file.

Step 71: End.

\subsection{Algorithm for function decryption_block(ct[],key[],forward_next,b ackward_next,ff[],bf[],block_size)}

Step-1: forward_next=forward_next+1

Step-2: forward_next=mod(forward_next,block_size)

Step-3: if forward_next=0 then forward_next=1 otherwise go to Step-4

Step-4: backward_next=backward_next+1

Step-5: backward_next=mod(backward_next,block_size)

Step-6: if backward_next=0 then backward_next $=1$ otherwise go to Step-7

Step-7: $(\mathrm{u}, \mathrm{v})=$ Call generateList (block_size, forward_next, backward next);

Step-8: initialise the array pt[block_size] with all zeros

Step-9: $\mathrm{k}=2 *$ block_size

Step-10: if k > block_size+1 then go to Step-45 otherwise go to Step-11

Step-11: $(\mathrm{i}, \mathrm{j})=$ Call whatIsIn $(\mathrm{u}[\mathrm{k}]$, block_size, forward_next ,backward_next, v[])

Step-12: if $\mathrm{i} !=\mathrm{j}$ then go to Step-13 otherwise Step-25

Step-13: if $i=0$ then go to Step-14 otherwise Step-15

Step-14: pos_i $=0$

Step-15: if v[Call oldPosition(i,block_size $)]=\mathrm{u}[\mathrm{k}]$ then go to Step-16 otherwise go to Step-17

Step-16: pos_i=Call oldPosition(i,block_size)

Step-17: pos_i=Call lastPosition(i,block_size)

Step-18: if $j=0$ the go to Step-19 otherwise go to Step-20 
Step-19: pos $\_$j $=0$

Step-20: if $\mathrm{v}($ Call oldPosition $(\mathrm{j}$, block_size $))=\mathrm{u}[\mathrm{k}]$ then go to Step-21 otherwise go to Step-22

Step-21: pos_j=Call oldPosition(j,block_size)

Step-22: pos_j=Call lastPosition(j,block_size)

Step-23: sub1=Call isChanged(i,pos_i)

Step-24: sub2=Call isChanged(j,pos_j) go to Step-27

Step-25: sub1=Call isChanged(i,oldPosition(i,block_size))

Step-26: sub2=Call isChanged(i,lastPosition(i,block_size))

Step-27: check1=ct[u[k]]-sub1-sub2-key[u[k]]

Step-28: if $i=0$ and $j=0$ then go to Step- 29 otherwise go to Step-34

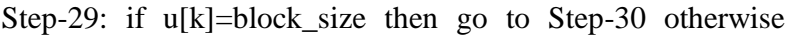
Step-31

Step-30: check=check1-bf

Step-31: if $\mathrm{u}[\mathrm{k}]=1$ then go to Step-32 otherwise go to Step-33

Step-32: check=check1-ff go to Step-39

Step-33: check=check1 go to Step-39

Step-34: if $\mathrm{u}[\mathrm{k}]=$ block_size and (Call conditionCheck $\left(\mathrm{u}[\mathrm{k}], \mathrm{i}, \mathrm{j}, \mathrm{block} \_\right.$size, $\left.\left.v\right)=1\right)$ then go to Step-35 otherwise go to Step-36

Step-35: check=check1-bf

Step-36: if $\mathrm{u}[\mathrm{k}]=1$ and (Call conditionCheck $(\mathrm{u}[\mathrm{k}], \mathrm{i}, \mathrm{j}$, block_size,v)=2) then go to Step-37 otherwise go to Step-38

Step-37: check=check1-ff

Step-38: check=check1

Step-39: if (check < 0) then go to Step-40 otherwise go to Step-42

Step-40: check=check+256

Step-41: go to Step-39

Step-42: check=mod(check,256)

Step-43: $\mathrm{pt}[\mathrm{u}[\mathrm{k}]]=$ check

Step-44: k=k+1 and go to Step-10

Step-45: return pt[] to the calling function

\subsection{Algorithm for function condition_check (number,i,j,block_size,v[])}

Step 1: if $i * j !=0$, go to step 2, else go to step 2,else go to step

Step 2: flag $=0$

Step 3: if $\mathrm{i} !=0$ go to Step 4,else go to step 8

Step 4: if v[Call oldPosition(i,block_size) $]==$ number, go to step 5, else go to step 6

Step 5: pos=Call oldPosition(i,block_size $)$

Step 6: pos=Call lastPosition(i,block_size)

Step 8: if v[Call oldPosition(j,block_size $)]==$ number, go to step 9 ,else go to step 10

Step 9: pos=Call oldPosition(j,block_size)

Step 10: pos=Call lastPosition(j,length)

Step 11: flag1=mod(pos,2)
Step 12: if flag1=0, go to step 13 ,else go to step 14

Step 13: flag=2

Step 14: flag=flag1

Step 15: Return flag to the calling function

\subsection{Algorithm for function is_changed (number,position,forward_next,backward next,block_size,u[],v[],ct[],ff[],bf[])}

Step 1: is number $==0$ ?

Step 2: if Step 1=TRUE, then go to sub=0 else go to Step 3

Step 3: if position=Call lastPosition(number,block_size) then $\mathrm{sub}=\mathrm{ct}$ [number] else go to Step 4

Step4: $[\mathrm{i}, \mathrm{j}] \quad$ Call whatIsInBetween (number,length,v,next1,next2)

Step 5: if i!=j go to step 6, else go to step 24

Step 6: if $i=0$ then set position_ $i=0$ else go to step 7

Step 7: if $\mathrm{v}[$ Call lastPosition(i,block_size) $]=$ number then set position_i=Call lastPosition(i,block_size) else go to Step 8

Step 8: set position_i=Call oldPosition(i,block_size)

Step 9: if $j==0$, set position $j=0$,else go to step 10

Step 10: if v[Call lastPosition(i,block_size)]=number, set position_j=Call lastPosition(j,block_size),else go to step 11

Step 11: set position_j=Call oldPosition(j,block_size)

Step12: $\quad$ sub1= ct[number]- isChanged (i,position_i,next1,next2,block_size,u,v,ct,ff,bf)isChanged(j,position_j,next1,next2,block_size,u,v,ct,ff,bf) Step13:[a,b]=Call whatIsIn (number,block_size,next1,next2, v)

Step 14: if $a=0$ and $b=0$ then go to step 15 ,else go to step 18

Step 15: if number=block_size,set $s u b=$ sub1+bf else go to step 16

Step 16: if number=1 then set sub=sub1+ff else go to step 17

Step 17: sub=sub1

Step18:flag= Call conditionCheck (number,a,b,block_size,v )

Step 19: if number=block_size and position=Call oldPosition(number) and flag!=1 go to Step 20, else go to step

Step 20: sub=sub1+bf

Step 21: if number=1 and position=Call oldPosition(number) and flag!=2 go to Step 22, else go to step 23

Step 22: sub=sub1+ff

Step 23: sub=sub1

Step24:sub1=ct[number]-isChanged

(i,oldPosition(i),next1,next2, block_size,u,v,ct,ff,bf)isChanged(i,lastPosition(i),next1,next2,block_size,u,v,ct,ff,bf)

Step 25: [a,b]=whatIsIn(number,block_size,next1,next2,v)

Step 26: if $a=0$ and $b=0$ then go to step 27,else go to step 30

Step 27: if number==block_size,set sub=sub1+bf else go to step 28

Step 28: if number==1, set sub=sub1+ff,else go to step 29

Step 29: sub=sub1

Step30: flag = Call conditionCheck (number,a,b,block_size,v) 
Step 31: if number==block_size and position==Call oldPosition(number,block_size) and flag!=1 go to Step 32, else go to step 33

Step 32: sub=sub1+bf

Step 33: if number $==1$ and position $==$ Call oldPosition(number,block_size) and flag!=2 go to Step 34, else go to step 35

Step 34: sub=sub1+ff

Step 35: sub=sub1

Step 36: Return sub to the calling function

\subsection{Algorithm for function what_is_in (number,block_size,forward_next,backwar d_next,v[])}

Step-1: if number+backward_next $<=$ block_size, then go to Step-2,otherwise Step-3

Step-2: i=number+backward_next

Step-3: i=number+backward_next-block_size,

Step-4: if number-forward_next $>=1$ then go to Step5,otherwise go to Step-6

Step-5: j=number-forward_next

Step-6: j=number-forward_next+block_size,

Step-7: lastPos_number $=$ Call lastPosition (number, block_size)

Step-8: if( $i=j$ and $i !=0)$ then go to Step-9,otherwise go to Step13

Step-9: if(Call lastPosition(i,block_size) $>$ lastPos_number) then go to Step-10,otherwise go to Step-11

Step-10: $i=0$

Step-11: if(Call oldPos(i,block_size)>lastPos_number) then go to Step-12,otherwise go to Step-23

Step-12: $\mathrm{j}=0$

Step-13: if(i!=0) then go to Step-14 otherwise go to Step-18

Step-14: if(Call lastPosition(i,block_size)>lastPos_number and $\mathrm{v}($ Call lastPosition(i,block_size $))=$ number $)$ then go to Step-15,otherwise go to Step-16

Step-15: i=0

Step-16: if(Call oldPos(i,block_size,)>lastPos_number and $\mathrm{v}($ Call oldPos(i,block_size, $))=$ number $)$ then go to Step17 ,otherwise go to Step-18

Step-17: $i=0$

Step-18: if(j!=0) then go to Step-19 otherwise go to Step-23

Step-19: if(Call lastPosition(j,block_size,)>lastPos_number and $\mathrm{v}($ Call lastPosition $(\mathrm{j}$, block_size, $))=$ number $)$ then go to Step-20,otherwise go to Step-21

Step-20: $j=0$

Step-21: if(Call oldPos(j,block_size)>lastPos_number and $\mathrm{v}($ Call oldPos(j,block_size $))=$ number $)$ then go to Step22,otherwise go to Step-23

Step-22: $j=0$

Step-23: Return $i$ and $j$ to the calling function

\subsection{Algorithm for function what_is_in_between (number,block_size,forward_next,backwar d_next,v[])}

Step-1: $\quad(\mathrm{i}, \mathrm{j}) \quad$ Call whatIsIn (number,block_size,,forward_next,backward_next,v[])

Step- 2 : if $i=j$ and $i !=0$ and $j !=0$ then go to Step-3,otherwise go to Step-10

Step-3: $\quad$ condition $=\left(\right.$ Call $\quad$ lastPosition $\left(i, b l o c k \_s i z e, ~\right)>$ Call oldPos(number,block_size, ) and Call lastPosition(i,block_size, $<$ Call

lastPosition(number,block_size, ) and $\mathrm{v}(\mathrm{Cal}$ lastPosition(i,block_size, $)$ )=number)

Step-4: if condition=0 then go to Step-5,otherwise go to Step6

Step-5: $i=0$

Step-6: $\quad$ condition $=($ Call oldPosition $(j$, block_size,$)>$ Call oldPosition(number,block size,) and Call oldPosition(j,block_size, $)<$ Call lastPosition(number,block_size,) and v(Call oldPosition(j,block_size, $))=$ number)

Step-7: if condition=0 then got to Step- 8 , otherwise go to Step-9

Step-8: $j=0$

Step-9: go to Step-20

Step-10: if $\mathrm{i} !=0$ then go to Step-11,otherwise go to Step-15

Step-11: condition $1=$ Call lastPosition(i,block_size, $)>$ Call oldPosition(number,block_size,) and Call lastPosition(i,block_size, $<$ Call

lastPosition(number,block_size, $) \quad$ and $\quad \mathrm{v}($ Call lastPosition(i,block_size, $)$ )=number

Step-12: $\quad$ condition2=Call oldPosition(i,block_size, $)>$ Call oldPos(number,block_size,) and Call oldPosition(i,block_size, $)<$ Call

lastPosition(number,block_size, ) and v(Call oldPosition(i,block_size, ))=number

Step-13: if condition $1=0$ and condition $=0$ then go to Step14 ,otherwise go to Step-15

Step-14: $i=0$

Step-15: if j!=0 then go to Step-16,otherwise go to Step-20

Step-16: condition $1=$ Call lastPosition $(j$, block_size, $)>$ Call oldPosition(number,block_size,) and Call lastPosition(j,block_size, $)<$ Call lastPosition(number,block_size, ) and v(Call lastPosition(j,block_size, $)$ )=number

Step-17: condition2=Call oldPosition $(\mathrm{j}$, block_size, $)>$ Call oldPosition(number,block_size,) and Call oldPosition(j,block_size, $)<$ Call

lastPosition(number,block_size, ) and v(Call oldPosition(j,block_size, $)$ )=number

Step-18: if condition $1=0$ and condition $=0$ then go to Step19,otherwise go to Step-20

Step-19: $\mathrm{j}=0$

Step-20: Return $\mathrm{i}$ and $\mathrm{j}$ to the calling function 


\subsection{Algorithm for function generate_list(block_size,forward_next,back ward_next)}

Step 1:-source=1.

Step 2:- i=1.

Step 3:-u[i]=source. $/ * \mathrm{u}$ contains the source of the Feedback Transfers.*/

Step 4:-if $(u[i]+\bmod ($ next, length $))>$ length, then $v[i]=u[i]+$ $\bmod ($ next,length) - length .

Step 5:- if $(\mathrm{u}[\mathrm{i}]+\bmod ($ next,length $))<=$ length, then $\mathrm{v}[\mathrm{i}]=\mathrm{u}[\mathrm{i}]+$ $\bmod ($ next,length).

Step 6:- source=source+1;

Step 7:- if $\mathrm{i}<(2 *$ length $)$; then $\mathrm{i}=\mathrm{i}+2$ and go to Step 3 .

Step 8:-source= length.

Step 9:- $\mathrm{i}=2$.

Step 10:-u[i]=source.

Step 11:-if $(u[i]-\bmod ($ next,length $))<1$, then $\quad v[i]=u[i]-$ $\bmod ($ next,length) + length.

Step 12:- if $(\mathrm{u}[\mathrm{i}]-\bmod ($ next,length $))>=1$, then $\mathrm{v}[\mathrm{i}]=\mathrm{u}[\mathrm{i}]$ $\bmod ($ next,length).

Step 13:- source=source-1;

Step 14:- if $\mathrm{i}<(2 *$ length); then $\mathrm{i}=\mathrm{i}+2$ and go to Step 10 .

Step 15:- Return Control to calling function, also return u[] and $\mathrm{v}[]$ to the calling function.

\subsection{Algorithm for function old_position(number,block_size)}

Step 1:-current_pos = Call last_Position_of (number, length);

Step 2:- first_pos $=2 *$ length - current_pos +1 ;

Step 3:-Return Control to calling function, and return first_pos to the calling function.

\subsection{Algorithm for function last_position(Number,Block_Size)}

Step 1:-if number <= ceil (length/2); go to Step 3

Step 2:- if number >ceil (length/2); go to Step 4

Step 3:-last_pos $=2 *$ length $-2 *($ number- 1$)$;'

Step 4:- last_pos $=2 *($ number- 1$)$;

Step 5:- Return Control to calling function,and return last_pos to the calling function.

\subsection{Algorithm for function pt_shift(num)}

Step 1:- seq=Call generate_sequence(num)

Step 2:-pt_bits=Call convertToBits(pt)

Step 3:-shifted_pt_bits=Bit_Rotation(pt,bits,seq[],0)

Step 4:- shifted_pt_bytes=convertToBytes(shifted_pt_bits)

Step 5:-Return shifted_pt_bytes to calling function.

\subsection{Algorithm for function ct_shift(ct[],num)}

Step 1:- seq=Call generate_sequence(num)

Step 2:-ct_bits=Call convertToBits(ct)
Step 3:-shifted_ct_bits=Bit_Rotation(ct,bits,seq[],1)

Step 4:- shifted_ct_bytes=convertToBytes(shifted_pt_bits)

Step 5:-Return shifted_ct_bytes to calling function.

\subsection{Algorithm for function} convert_to_bits(a[])

Step 1:- k=1

Step 2:- i=1 to length of a[] Step=1 do

Step $3:-\mathrm{j}=8$ to 1 step $=-1$ do

Step 4:-aux[k]=Call bitget(a[i],j)

Step 5:=k=k+1;

Step 6:-If $j>1$ then go to Step 4 else go to Step 7

Step 7:-If $\mathrm{i}<$ length of a[] then go to Step 3 else go to Step 8 Step 8:-Return aux[] to the calling function.

\subsection{Algorithm for function convert_to_bytes(a[])}

Step 1:- k=1

Step 2:- $\mathrm{i}=1$ to (length of a[])/8 Step=1 do

Step 3:-sum $=0$

Step 4:- $\mathrm{j}=7$ to 0 step $=-1$ do

Step 5:-sum $=$ sum $+\mathrm{a}[\mathrm{k}] * 2^{\wedge} \mathrm{j}$

Step 6:-k=k+1

Step 7:-If $\mathrm{j}>0$ then go to Step 5 else go to Step 8

Step 8:-b[i]=sum

Step 9:-If $\mathrm{i}<($ length of $\mathrm{a}[\mathrm{l}) / 8$ then go to Step 3 else go to Step 10

Step 10:-Return $b[]$ to the calling function.

\subsection{Algorithm for function generate_sequence(num)}

This function simply generates a sequence array according to the generated keypad in order to make sure that the bits are rotated in a dynamic fashion rather than in the same way every round, which would render the rotation of bits impractical.

\subsection{Algorithm for function} bit_rotation(b[],seq[],flag)

Step 1:- len= length of b[] array

Step 2:- n=Integral part of square root of len.

Step 3:-Array a[n][n] is filled row-major wise with the bits in $\mathrm{b}[]$ array.

Step 4:-If flag=1(signifying Decryption), the seq[] array is reversed.

Step 5:- The 24 different bit shifting functions are called in a sequence given by the seq[] array.

Step 6:- Jumbled bits are copied back into the $b[]$ array.

Step 7:- b[] array is returned to the calling function.

\subsection{1) Diagonal1_Down_Shift}

In this function, the major diagonal is shifted one place downwards, the shifting being cyclic.

2.18.2) Diagonal2_Down_Shift 
In this function, the second diagonal is shifted one place downwards, the shifting being cyclic.

\subsection{3) Diagonal1_Up_Shift}

In this function, the major diagonal is shifted one place upwards, the shifting being cyclic.

\subsection{4) Diagonal2_Up_Shift}

In this function, the second diagonal is shifted one place upwards, the shifting being cyclic.

\subsection{5) Exchange_Diagonals_ColumnWise}

In this function, the two diagonals in the bit matrix are exchanged with each other column wise.

\subsection{6) Exchange_Diagonals_RowWise}

In this function, the two diagonals in the bit matrix are exchanged with each other row wise.

\subsection{7) Flip_Diagonal1}

In this function, the order of the major diagonal elements is reversed.

\subsection{8) Flip_Diagonal2}

In this function, the order of the second diagonal elements is reversed.

\subsection{9) Up_Shift_Even}

In this function, Even rows are shifted upwards by one.

\subsubsection{0)Down_Shift_Even}

In this function, Even rows are shifted downwards by one.

\subsubsection{1)Up_Shift_Odd}

In this function, Odd rows are shifted upwards by one.

\subsubsection{2)Down_Shift_Odd}

In this function, Odd rows are shifted downwards by one.

\subsubsection{3)Exchange_Even_Column}

In this function, Even columns are exchanged with each other.

\subsubsection{4)Exchange_Odd_Column}

In this function, Odd columns are exchanged with each other.

\subsubsection{5)Exchange_Even_Row}

In this function, Even rows are exchanged with each other.

\subsubsection{6)Exchange_Odd_Row}

In this function, Odd rows are exchanged with each other.

\subsubsection{7)Left_Shift_Even}

In this function, Even rows are shifted one place to the left.

\subsubsection{8)Left_Shift_Odd}

In this function, Odd rows are shifted one place to the left.

\subsubsection{9)Right_Shift_Even}

In this function, Even rows are shifted one place to the right.

\subsubsection{0)Right_Shift_Odd}

In this function, Odd rows are shifted one place to the right.

\subsubsection{1)Rotate_Even_AntiClockwise}

In this function, Even interior circles are rotated AntiClockwise.

\subsubsection{2)Rotate_Odd_AntiClockwise}

In this function, Odd interior circles are rotated AntiClockwise.

\subsubsection{3)Rotate_Even_Clockwise}

In this function, Even interior circles are rotated Clockwise.

\subsubsection{4)Rotate_Odd_Clockwise:}

In this function, Odd interior circles are rotated Clockwise.

\section{RESULTS AND DISCUSSIONS}

\subsection{Encryption of small plain texts with given seed}

In the table given on the Left Hand Side of the next page (Table-I), there are many instances where it is observed that for the same seed, almost similar Plain Texts in SL. NO 1, 2 and 3, NO. 4,5 and 6, NO. 9 and 10, the Cipher Texts are totally haphazard thus rendering the Plain Text irretrievable. Therefore, for slightly bigger Plain Texts the retrieval of the Plain Text becomes almost impossible for any machine as well unless the key, i.e. seed is known.

Table-1. Small test cases

\begin{tabular}{|c|c|c|c|}
\hline $\begin{array}{l}\text { SL. } \\
\text { NO. }\end{array}$ & $\begin{array}{l}\text { PLAIN } \\
\text { TEXT }\end{array}$ & SEED & CIPHER TEXT \\
\hline 1 & AAAAAA & Xaviers & $3(! @ \cdot \tilde{n}$ \\
\hline 2 & BAAAAA & Xaviers & -'»IL_ \\
\hline 3 & CAAAAA & Xaviers & 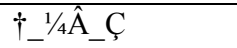 \\
\hline 4 & $\begin{array}{l}\mathrm{ABABAB} \\
\mathrm{A}\end{array}$ & Xaviers & Ú'\#² $\left.{ }^{2} \_\right\} \ddot{y}$ \\
\hline 5 & $\begin{array}{l}\text { ACACAC } \\
\text { A }\end{array}$ & Xaviers & __I.ay \\
\hline 6 & $\begin{array}{l}\mathrm{BCBCBC} \\
\mathrm{B}\end{array}$ & Xaviers & $\begin{array}{l}\circledR \S \mathrm{d} \\
\text { ¿z7 }\end{array}$ \\
\hline 7 & $\begin{array}{l}\text { AAABAA } \\
\text { A }\end{array}$ & Xaviers & ðš7õ $f 4 Y ́$ \\
\hline 8 & $\begin{array}{l}\text { AAACAA } \\
\text { A }\end{array}$ & Xaviers & ]MŸa'ý_ \\
\hline 9 & $\begin{array}{l}\text { AABAAB } \\
\text { A }\end{array}$ & Xaviers & _J Jaš_ \\
\hline 10 & $\begin{array}{l}\text { AACAAC } \\
\text { A }\end{array}$ & Xaviers & $8 \mathrm{É} 1 ß Y \mu 0$ \\
\hline
\end{tabular}




\subsection{Encryption of a paragraph with given seed}

In the encrypted Cipher Text shown on the Right Hand Side, a better example of the efficiency of the algorithm can be observed since the size of each block in every iteration of the encryption process plays an important role in completely diffusing the Plain Text into a seemingly random and completely incomprehensible Cipher Text.

Table 2.Encryption of a paragraph with seed Xaviers

\begin{tabular}{|c|c|}
\hline Plain text & Cipher text \\
\hline $\begin{array}{l}\text { St. Xavier's has always } \\
\text { been known for his } \\
\text { cosmopolitan and } \\
\text { national character. Much } \\
\text { before the expression } \\
\text { "national integration" } \\
\text { gained currency, St. } \\
\text { Xavier's had tried to } \\
\text { foster among its students } \\
\text { the spirit and practice of } \\
\text { it. Coming as they do } \\
\text { from all over India and } \\
\text { from various } \\
\text { communities, they live } \\
\text { in complete harmony, } \\
\text { understanding and } \\
\text { mutual respect. Thus } \\
\text { they are encouraged to } \\
\text { develop beyond local } \\
\text { and group affinities, } \\
\text { loyalties to the country } \\
\text { and the society at large. }\end{array}$ & 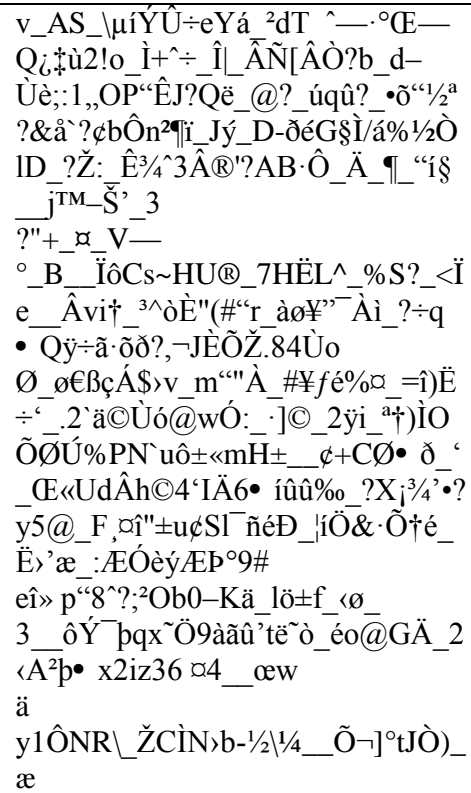 \\
\hline
\end{tabular}

\subsection{Graphs and frequency analysis charts}

The graphs given below give us a good estimate of the randomness of the occurrence frequencies of the different ASCII characters. In the ASCII ' 0 ', ASCII ' 1 ' charts varied results are obtained even when the same character is encrypted over and over again.

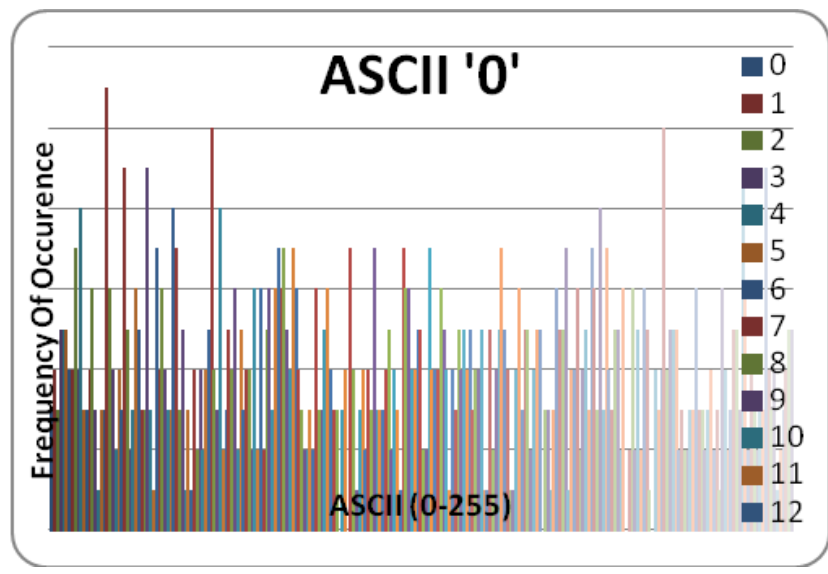

Fig 1: Frequency spectral graph for ASCII '0' for AFES Version-1

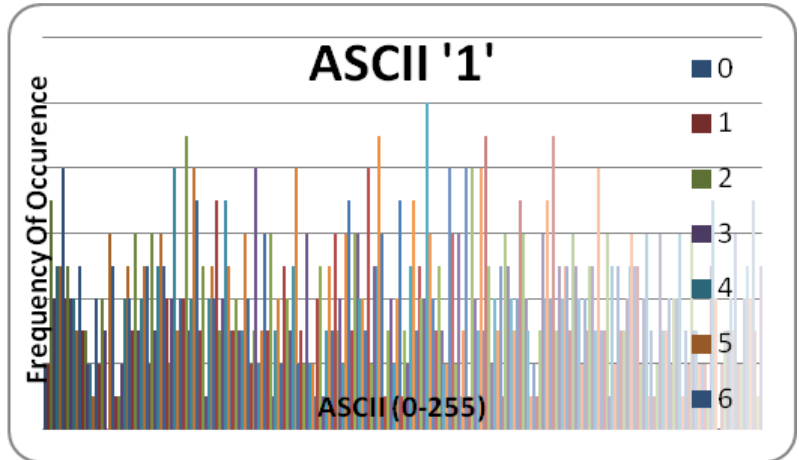

Fig 2: Frequency spectral graph for ASCII '1' for AFES Version-1

\section{CONCLUSION AND FUTURE SCOPE}

The present method has been tested on various types of files such as .doc, .jpg, .bmp, .exe, .com, .dbf, .wav, .avi and the results were quite satisfactory. The encryption and decryption methods work smoothly. In the present method the encrypted text cannot be decrypted without knowing the exact initial keypad. The results show that, the set of strings where there is a difference in only one character in the plain text, the encrypted texts are coming totally different from each other. The present method is free from any kind of brute force attack or known plain text attack. The present AFES Ver-1 may be applied to encrypt any short message, password, confidential key and even images and other file types as well. One can apply this method to encrypt data in sensor networks as well.

\section{ACKNOWLEDGMENTS}

The authors are extremely grateful to the Department of Computer Science for providing the opportunity to work on Symmetric Key Cryptography. A.N sincerely expresses his gratitude to Fr. Dr. Felix Raj, Principal of St. Xavier's College (Autonomous) for giving constant encouragement regarding research in the field of Cryptography.

\section{REFERENCES}

[1] Purnendu Mukherjee, Prabal Banerjee, AsokeNath, "Multi Way Feedback Encryption Standard VerI(MWFES-I)", International Journal of Advanced Computer Research(IJACR), Volume-3, Number-3, Issue-11, September 2013, Pages:176-182.

[2] Prabal Banerjee, Purnendu Mukherjee, AsokeNath, "Modified Multi Way Feedback EncryptionStandard : Ver-I (MMWFES-I)", International Journal of Advanced Computer Research(IJACR),Vol-3, No.1, Issue-13, Page 352-360, Dec(2013).

[3] Asoke Nath, Debdeep Basu, Ankita Bose, Saptarshi Chatterjee, Surajit Bhowmik," "Multi Way Feedback Encryption Standard Ver-2(MWFES-2)," International Journal of Advanced Computer Research(IJACR), Vol-3, Number-1, Issue-13, Page-29-35, Dec(2013).

[4] Saptarshi Chatterjee, Debdeep Basu, Ankita Bose, Surajit Bhowmik, Asoke Nath," "Modified Multi Way Feedback Encryption Standard Ver-2(MMWFES-2)," JGRCS, Vol-4, No. 12, December, 2013, Page 8-13(2013).

[5] Asoke Nath, Debdeep Basu, Ankita Bose, Saptarshi Chatterjee and Surajit Bhowmik "Multi Way Feedback Encryption Standard Ver-3(MWFES-3)," published in IEEE conference proceedings: WICT-2013 held at Hanoi in Dec 14-18(2013), page 318-325(2013). 
[6] Asoke Nath, Payel Pal, "Modern Encryption Standard Ver-IV(MES-IV)," International Journal of Advanced Computer Research(IJACR), Volume-3, Number-3, Issue-11, September 2013, Page:216-223.

[7] Asoke Nath, Bidhusundar Samanta, "Modern Encryption Standard Ver-V(MES-V)," International Journal of Advanced Computer Research(IJACR), Volume-3, Number-3, Issue-11, September 2013, Pages:257-264.

[8] Asoke Nath, Saima Ghosh, Meheboob Alam Mallik,"Symmetric Key Cryptography using Random Key generator" Proceedings of International conference on security and management(SAM '10) held at Las Vegas, USA July 12-15, 2010), Vol-2, Page: 239244(2010).

[9] Dripto Chatterjee, Joyshree Nath, Soumitra Mondal, Suvadeep Dasgupta, Asoke Nath, "Advanced Symmetric key Cryptography using extended MSA method: DJSSA symmetric key algorithm", Journal of Computing, Vol 3, Issue-2, Page 66-71,Feb(2011).

[10] Dripto Chatterjee, Joyshree Nath, Suvadeep Dasgupta and AsokeNath, "A new Symmetric key Cryptography Algorithm using extended MSA method: DJSA symmetric key algorithm," Proceedings of IEEE International Conference on Communication Systems and Network Technologies, held at SMVDU(Jammu) 0306 June,2011, Page-89-94(2011).

[11] Neeraj Khanna, Joel James, Joyshree Nath, Sayantan Chakraborty, Amlan Chakrabarti, Asoke Nath, "New Symmetric key Cryptographic algorithm using combined bit manipulation and MSA encryption algorithm: NJJSAA symmetric key algorithm", Proceedings of IEEE CSNT-2011 held at SMVDU(Jammu) 03-06 June 2011, Page 125-130(2011)

[12] Dripto Chatterjee, Joyshree Nath, Sankar Das, Shalabh Agarwal, Asoke Nath, "Symmetric key Cryptography using modified DJSSA symmetric key algorithm", Proceedings of International conference Worldcomp 2011 held at Las Vegas 18-21 July 2011, Page-306-311, Vol-1(2011).

[13] Debanjan Das, Joyshree Nath, Megholova Mukherjee, Neha Chaudhury and Asoke Nath, "An Integrated symmetric key cryptography algorithm using generalized vernam cipher method and DJSA method: DJMNA symmetric key algorithm", Proceedings of IEEE International conference: World Congress WICT-2011 held at Mumbai University 11-14 Dec, 2011, Page No.1203-1208(2011).

[14] Trisha Chatterjee, Tamodeep Das, Joyshree Nath, Shayan Dey and Asoke Nath, "Symmetric key cryptosystem using combined cryptographic algorithmsgeneralized modified vernam cipher method, MSA method and NJJSAA method: TTJSA algorithm", Proceedings of IEEE International conference: World Congress WICT-2011 theld at Mumbai University 11-14 Dec, 2011, Page No. 1179-1184(2011).
[15] Trisha Chatterjee, Tamodeep Das, Shyan Dey, Joyshree Nath and Asoke Nath,Symmetric key Cryptography using two-way updated Generalized Vernam Cipher method: TTSJA algorithm, International Journal of Computer Applications (IJCA, USA), Vol 42, No.1, March, Pg: 34 -39( 2012).

[16] Satyaki Roy, Navajit Maitra, Joyshree Nath, Shalabh Agarwal and Asoke Nath, "Ultra Encryption Standard(UES) Version-I: Symmetric Key Cryptosystem using generalized modified Vernam Cipher method, Permutation method and Columnar Transposition method", Proceedings of IEEE sponsored National Conference on Recent Advances in Communication, Control and Computing Technology -RACCCT 2012, 29-30 March held at Surat, Page 81-88(2012).

[17] Somdip Dey, Joyshree Nath, Asoke Nath, “An Integrated Symmetric Key Cryptographic Method - Amalgamation of TTJSA Algorithm, Advanced Caeser Cipher Algorithm, Bit Rotation and reversal Method: SJA Algorithm", International Journal of Modern Education and Computer Science, (IJMECS), ISSN: 2075-0161 (Print), ISSN: 2075-017X (Online), Vol-4, No-5, Page 19,2012 .

[18] Somdip Dey, Joyshree Nath, Asoke Nath, “An Advanced Combined Symmetric Key Cryptographic Method using Bit manipulation, Bit Reversal, Modified Caeser Cipher(SD-REE), DJSA method, TTJSA method: SJA-I Algorithm," International Journal of Computer Applications(IJCA 0975-8887, USA), Vol. 46, No.20, Page- 46-53,May, 2012.

[19] Satyaki Roy, Navajit Maitra, Joyshree Nath, Shalabh Agarwal and Asoke Nath, "Ultra Encryption Standard(UES) Version-IV: New Symmetric Key Cryptosystem with bit-level columnar Transposition and Reshuffling of Bits", International Journal of Computer Applications(IJCA)(0975-8887) USA Volume 51No.1.,Aug, Page. 28-35(2012)

[20] Neeraj Khanna, Dripto Chatterjee, Joyshree Nath and Asoke Nath, "Bit Level Encryption Standard(BLES) : Version-I," International Journal of Computer Applications(IJCA)(0975-8887) USA Volume 52No.2.,Aug, Page.41-46(2012).

[21] Prabal Banerjee, Asoke Nath, "Bit LevelGeneralized Modified Vernam Cipher Method with Feedback", Proceedings of International Conference on Emerging Trends and Technologies held at Indore, Dec 15-16, 2012.

[22] Prabal Banerjee, Asoke Nath, "Advanced Symmetric Key cryptosystem using Bit and Byte Level encryption methods with Feedback", Proceedings of International conference Worldcomp 2013 held at Las Vegas, July 2013.

[23] Ankita Bose, Debdeep Basu, Saptarshi Chatterjee, Asoke Nath, Surajit Bhowmik, "Bit Level Multi Way Feedback Encryption Standard Version-1(BLMWFES-1)", Paper published at IEEE CSNT 2014 held at Bhopal 7-9 April. 